\title{
COHOMOLOGY RELATIONS IN SPACES WITH A TOPOLOGICAL TRANSFORMATION GROUP ${ }^{1)}$
}

\author{
SZE-TSEN HU
}

\section{Introduction}

Let $Q$ be a topological transformation group operating on the left of a topological space $X$. Let us denote by. $B$ the orbit space and $p: X \rightarrow B$ the projection. $p$ is a continuous and open map of $X$ onto $B$. For an arbitrary abelian coefficient group $G$, the continuous map $p$ induces homomorphisms

$$
p^{*}: H^{n}(B, G) \rightarrow H^{n}(X, G), \quad(n \geq 0),
$$

of the Alexander-Wallace cohomolog groups $[1]^{2)}$. These induced homomorphisms are, in general, not onto isomorphisms. They depend on the manner in which the topological transformation group $Q$ operates on $X$.

To measure the deviation of these induced homomorphisms $p^{*}$ from the onto isomorphisms. we introduce, in the present paper, the weakly residual coho:nology groups

$$
H_{w}^{n}(X, G), \quad(n \geq 0) .
$$

They are invariants depending on $X, Q, G$ and the operations of $Q$ on $X$. By means of these groups. we shall establish an exact sequence

$$
H^{0}(B, G) \stackrel{p^{*}}{\rightarrow} \ldots \rightarrow H^{n}(B, G) \stackrel{p^{*}}{\rightarrow} H^{n}(X, G) \rightarrow H_{w}^{n}(X, G) \rightarrow H^{n+1}(B, G) \stackrel{p^{*}}{\rightarrow} \ldots
$$

This indicates that the weakly residual cohomolegy groups $H_{w}^{n}(X, G)$ might play an important role in the further studies of the cohomology structures of the orbit space.

For each point $x \in X$, there is a canonical homomorphism

$$
k_{x}^{*}: H_{w}^{\prime \prime}(X, G) \rightarrow H^{n}(Q, G), \quad(n \geqslant 0) .
$$

It is proved that if $Q$ is compact and if $x$ and $y$ are two points contained in a compact connected subset of $X$ then $k_{x}^{*}=k_{y}^{*}$.

\section{Preliminaries}

Throughout the present paper, let $Q$ be a topological group acting as a

Received, August 10, 1952.

i) Presented to the American Mathematical Society, September 2, 1952. This work was done under Contract N7-ONR-424, Office of Naval Research.

2) Numbers in square brackets refer to the bibliography at the end of the paper. 
group of transformations on the left of a topological space $X$. By this we mean that, with each element $q$ in $Q$, there is associated a transformation

$$
W_{q}: X \rightarrow x
$$

such that, if we use the notation $W_{q}(x)=q x$, the following conditions are satisfied.

$$
q x \text { is continuous in } q \text { and } x \text { simultaneously; }
$$$$
q_{1}\left(q_{2} x\right)=\left(q_{1} q_{2}\right) x, \quad\left(q_{1} \in Q, q_{2} \in Q, x \in X\right) ;
$$

$$
e x=x, \quad(x \in X),
$$

where $e$ denotes the neutral element of $Q$. More precisely, the condition (2.1) means that the map

$$
M: Q \times X \rightarrow X
$$

delined by $M(q, x)=q x$ for each $q \in Q$ and each $x \in X$ is continuous. Obviously, $W_{q}$ is a homeomorphism of $X$ for each $q \in Q$.

Two points $x$ and $y$ are said to be equivalent if there exists an element $q$ in $Q$ such that $y=q x$. This equivalence relation divides the points of $X$ into disjoint equivalence classes called the orbits of $Q$ in $X$. The orbit which contains the point $x \in X$ will be denoted by $Q x$. Hence $Q x=Q y$ if and only if $x$ and $y$ are equivalent. Let $B$ denote the set of all orbits of $Q$ in $X$. There is a natural map

$$
p: X \rightarrow B
$$

of $X$ onto $B$ defined by $p(x)=Q x$ for each $x \in X$. $p$ will be called the projection of $X$ onto $B$. Let us give $B$ the identification topology determined by $p$. That is to say, a subset $V$ in $B$ is called open if and only if $p^{-1}(V)$ is an open set in $X$. The topological space $B$ thus obtained will be called the orbit space of the transformation group $Q . \quad B$ is a $T_{1}$-space if and only if every orbit of $Q$ is a closed subset in $X$.

The projection $p: X \rightarrow B$ is both continuous and open. In fact, the continuity of $p$ follows from the definition of the identification topology in $B$ determined by $p$. To see that $p$ is open, let $U$ be an arbitrary open set in $X$ and call $V=p(U)$. It suffices to show that $p^{-1}(V)$ is an open set in $X$. By the definition of $p$, the set $p^{-1}(V)$ consists of the totality of the points $q x$ in $X$ such that $q \in Q$ and $x \in U$. Hence $p^{-1}(V)$ is the union $Q U$ of the sets $W_{q}(U)$ for all $q \in Q$. For each $q$ in $Q, W_{q}$ is a homeomorphism of $X$. This implies that $W_{q}(U)$ is open and hence, as a union of open sets, $p^{-1}(V)$ is open.

\section{The various cohomology groups}

For convenience of the reader, we shall briefly recall the definition of the 
Alexander-Wallace cohomology groups [1]. Let $G$ be an abelian group used as the coefficient group of the various cohomology groups defined in the sequel.

Denote by

$$
A^{n}(X, G), \quad(n \geqslant 0),
$$

the group of all $n$-functions $\phi: X^{n+1} \rightarrow G$ on $X$ into $G$ and

$$
A_{0}^{n}(X, G), \quad(n \geqslant 0),
$$

the subgroup of $A^{n}(X, G)$ consisting of the $n$-functions with empty support, where the support $S(\phi)$ of an $n$-function $\phi: X^{n+1} \rightarrow G$ is the closed set of $X$ defined by the following assertion:

(3.1) A point $x \in X$ is not in $S(\phi)$ if and only if there exists an open neighborhood $U$ of $x$ in $X$ such that

$$
\phi\left(x_{0}, x_{1}, \ldots, x_{n}\right)=0
$$

whenever $x_{i} \in U$ for all $i=0,1, \ldots, n$.

The coboundary homomorphism

$$
\delta: A^{n}(X, G) \rightarrow A^{n+1}(X, B)
$$

is defined as usual, namely ${ }^{3)}$

$$
(\delta \phi)\left(x_{0}, \ldots, x_{n+1}\right)=\sum_{i=0}^{n+1}(-1)^{i} \phi\left(x_{0}, \ldots, \hat{x}_{i}, \ldots, x_{n+1}\right)
$$

for arbitrary $\left(x_{0}, \ldots, x_{n+1}\right) \in X^{n+2}$. Obviously we have

$$
\delta\left(A_{0}^{n}((X, G)) \subset A_{0}^{n+1}(X, G) .\right.
$$

Let

$$
C^{n}(X, G)=A^{n}(X, G) / A_{0}^{n}(X, G) .
$$

Then $\delta$ in (3.2) induces a coboundary homomorphism

$$
\delta: C^{n}(X, G) \rightarrow C^{n+1}(X, G) .
$$

The elements of $C^{n}(X, G)$ are called the $n$-cochains of $X$ over $G$. For each $n$-function $\psi \in A^{n}(X, G)$, we shall denote by $[\phi]$ the $n$-cochain which contains $\phi$, that is,

$$
[\phi]=\phi+A_{0}^{n}(X, G),
$$

We say that $\phi$ represents $[\phi]$.

Let $Z^{n}(X, G) \subset C^{n}(X, G)$ denote the kernel of $\delta$ in (3.3), and $B^{n+1}(X, G)$ $=\delta\left(C^{n}(X, G)\right)$. Further, we define $B^{0}(X, G)=0$. Since $\delta \delta=0$, we have

The quotient group

$$
\begin{aligned}
& B^{\eta}(X, G) \subset Z^{n}(X, G), \quad(n \geqslant 0) . \\
& H^{n}(X, G)=Z^{n}(X, G) / B^{n}(X, G)
\end{aligned}
$$

3) The circumflex over $x_{i}$ indicates that $x_{i}$ is omitted. 
is called the $n$-dimensional cohomology group of $X$ over $G$.

An $n$-function $\phi \in A^{n}(X, G)$ is said to be strongly invariant under $Q$ if

$$
\phi\left(q_{0} X_{0}, \ldots q_{n} x_{n}\right)=\phi\left(x_{i}, \ldots, x_{n}\right)
$$

for all $x_{i} \in X$ and all $q_{i} \in Q, i=0, \ldots n$. An $n$-cochain $c \in C^{n}(X, G)$ is said to be strongly invariant under $Q$ if $c$ contains an $n$-function $\phi \in A^{\lambda}(X, G)$ which is strongly invariant under $Q$. Obviously the strongly invariant $n$-cochains of $X$ over $G$ form a subgroup

and

$$
C_{s}^{n}(X, G) \subset C^{n}(X, G)
$$

$$
\delta\left(C_{s}^{n}(X, G)\right) \subset C_{s}^{n+1}(X, G),
$$

hence the $o$ in (3.3) defines a coboundary homomorphism

$$
o: C_{s}^{n}(X, G) \rightarrow C_{s}^{n-1}(X, G) \text {. }
$$

Let $Z_{s}^{\prime \prime}(X, G) \subset C_{s}^{n}(X, G)$ denote the kernel of $\delta$ in (3.4) and $B_{s}^{n+1}(X . \mathrm{G})$ $=\delta\left(C_{s}^{n}(X, G)\right)$. Further, we define $B_{s}^{0}(X, G)=0$. Then evidently we have

$$
Z_{s}^{\prime \prime}(X, G)=Z^{n}(X, G) \cap C_{s}^{n}(X, G) .
$$

The quotient group

$$
H_{s}^{n}(X, G)=Z_{s}^{n}(X, G) / B_{s}^{n}(X, G)
$$

is called the $n$-dimensional strongly invariant cohomology group of $X$ over $G$ (under the topological transformation group $Q$ ).

For each integer $n \geq 0$, let

$$
C_{w}^{n}(X, G)=C^{n}(X, G) / C_{s}^{n}(X, G) .
$$

The elements of $C_{w}^{n}(X, G)$ are called the weakly residual n-cochains (with respect to $Q$ ) of $X$ over $G$. Since the coboundary homomorphism $o$ in (3.3) maps $C_{s}^{n}(X, G)$ into $C_{s}^{r+i}(Y, G)$, it induces a coboundary homomorphism

$$
o: C_{w}^{n}(X, G) \rightarrow C_{w}^{n+1}(X, G) \text {. }
$$

Let $Z_{w}^{n}(X, G) \subset C_{w}^{n}(X, G)$ denote the kernel of $o$ in $(3.5)$ and $B_{w}^{n+1}(X, G)$ $=\delta\left(C_{w}^{\prime \prime}(X, G)\right)$. Further, we define $B_{w}^{0}\left(N_{2}^{n}, G\right)=0$. The quotient group

$$
H_{w}^{n}(X, G)=Z_{w}^{n}(X, G) / B_{v}^{n}(X, G)
$$

is called the $n$-dimensional weakly residual cohomology group of $X$ over $G$ (with respect to the topological transformation group $Q$ ).

Let us denote respectively by

$$
\begin{aligned}
& :: C_{s}^{n}(X, G) \rightarrow C^{n}(X, G), \\
& \pi: C^{n}(X, G) \rightarrow C_{w}^{n}(X, G)
\end{aligned}
$$


the natural inclusion and projection homomorphisms. Since both : and $\pi$ commute with the coboundary operator $\delta$. they induce homomorphisms

$$
\begin{aligned}
\iota^{*}: H_{s}^{n}(X, G) & \rightarrow H^{n}(X, G), \\
\pi^{*}: H^{n}(X, G) & \rightarrow H_{w}^{n}(X, G)
\end{aligned}
$$

for each integer $n \geqslant 0$. We are going to define a homomorphism

$$
\delta^{*}: H_{w}^{n}(X, G) \rightarrow H_{s}^{n+1}(X, G)
$$

for every $n \geqslant 0$ as follows. Let $\alpha$ be an arbitrary element of $H_{w}^{n}(X, G)$. Choose a weakly residual $n$-cocycle $c_{w} \in C_{w}^{n}(X, G)$ which represents $\alpha$. Since $\pi$ maps $C^{n}(X, G)$ onto $C_{w}^{n}(X, G)$, there is an $n$-cochain $c \in C^{n}(X, G)$ with $\pi c=c_{w}$. Since $\pi \delta c=\delta \pi c=\delta c_{u^{\prime}}=0$, we have $\delta c \in Z_{s}^{n+1}(X, G)$. Hence $\delta c$ represents an element $\beta$ of $H_{s}^{n+1}(X, G)$. It is not dificult to see that, 3 depends only on $\alpha$. We define the homomorphism $\delta^{*}$ by taking

$$
\delta^{*}(\alpha)=\beta \text {. }
$$

The following theorem is a direct consequence of a general theorem of Kelley and Pitcher [2].

THEOREM I. The sequence of groups and homomorphisms

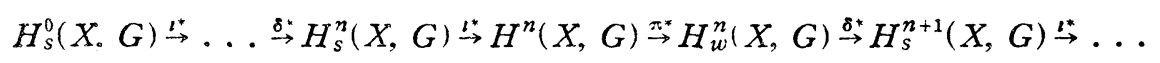

is exact in the sense that the image of each homomorphism coincides with the kernel of the following one.

\section{The isomorphism $p_{s}^{*}$}

The projection $p: X \rightarrow B$ induces a homomorphism

$$
p^{*}: A^{n}(B, G) \rightarrow A^{n}(X . G)
$$

of the $n$-functions $A^{n}(B, G)$ of the orbit space $B$ into the $n$-functions $A^{n}(X, G)$ of $X$ as follows. Let $\phi \in A^{n}(B . G)$ be an arbitrarily given $n$-functions of the orbit space $B$ into $G$. The $n$-function $p^{*} \phi \in A^{n}(X, G)$ is defined by

$$
\left(p^{*} \phi\right)\left(x_{i,}, \ldots, x_{i n}\right)=\phi\left(p x_{v}, \ldots, p x_{n}\right)
$$

for every $\left(x_{0} \ldots, x_{n}\right)$ of $X^{n+1}$. Since

$$
p(q x)=p(x)
$$

for every $x \in X$ and every $q \in Q, p^{\#} \phi$ is strongly invariant under $Q$. Let us denote by

$$
A_{S}^{n}(X . G)
$$

the subgroup of $A^{n}(X, G)$ which consists of the strongly invariant $n$-functions. Then (4.1) may be written in the following more precise form 


$$
p_{s}^{\sharp}: A^{n}(B, G) \rightarrow A_{s}^{n}(X, G) .
$$

(4.3) Lemma. pps maps $A^{n}(B, G)$ isomorphically onto $A_{s}^{n}(X, G)$.

Proof. That $p_{s}^{\#}$ is an isomorphism is a consequence of the fact that $p$ is onto. In fact, suppose that $\phi \in A^{n}(B, G)$ and $p_{s}^{\#} \phi=0$. Let $\left(b_{0}, \ldots, b_{n}\right)$ be an arbitrary point of $B^{n+1}$. Since $p$ maps $X$ onto $B$, there are $n+1$ points $x_{0}, \ldots$, $x_{n}$ in $X$ such that $p x_{i}=b_{i}$ for each $i=0, \ldots, n$. Then we have

$$
\phi\left(b_{0}, \ldots, b_{n}\right)=\left(p_{s}^{*} \phi\right)\left(x_{0}, \ldots, x_{n}\right)=0 .
$$

Since $\left(b_{0}, \ldots, b_{n}\right)$ is arbitrary, this proves that $\phi=0$ and hence $p_{s}^{\#}$ is an isomorphism.

To prove that $p_{s}^{\sharp}$ maps $A^{n}(B, G)$ onto $A_{s}^{n}(X, G)$, let

$$
\psi: X^{n+1} \rightarrow G
$$

be an arbitrary strongly invariant $n$-function. Define an $n$-function

$$
\phi: B^{n+1} \rightarrow G
$$

as follows. Let $\left(b_{0}, \ldots, b_{n}\right)$ be any point in $B^{n+1}$. Choose $n+1$ points $x_{0}$, $\ldots, x_{n}$ in $X$ such that $p x_{i}=b_{i}$ for each $i=0, \ldots, n$. Then $\phi$ is defined by taking

$$
\phi\left(b_{0}, \ldots, b_{n}\right)=\psi\left(x_{0}, \ldots, x_{n}\right) .
$$

To justify this definition, it suffices to show that $\phi\left(b_{0}, \ldots, b_{n}\right)$ does not depend on the choice of $x_{0}, \ldots, x_{n}$. In fact, let $y_{0}, \ldots, y_{n}$ be any $n+1$ points in $X$ with $p y_{i}=b_{i}$ for each $i=0, \ldots, n$. Then there are $q_{0}, \ldots, q_{n}$ in $Q$ such that

$$
y_{i}=q_{i} x_{i}, \quad(i=0, \ldots, n) .
$$

It follows from the strong invariance of $\psi$ that

$$
\psi\left(y_{0}, \ldots, y_{n}\right)=\psi\left(q_{0} x_{0}, \ldots, q_{n} x_{n}\right)=\psi\left(x_{0}, \ldots, x_{n}\right) .
$$

This justifies the definition of $\phi$. By (4.4), it is clear that $\phi=p_{s}^{*} \phi$. Hence $p_{s}^{\#}$ maps $A^{n}(B, G)$ onto $A_{s}^{n}(X, G)$. This completes the proof of (4.3).

(4.5) Lemma. $p_{s}^{\#}$ maps $A_{0}^{n}(B, G)$ onto $A_{s}^{n}(X, G) \cap A_{0}^{n}(X, G)$.

Proof. Let $\phi \in A_{0}^{n}(B, G)$ and $x \in X$ be arbitrarily given. Call $b=p x$. Since $\phi$ is of empty support, there is an open neighborhood $V$ of $b$ in $B$ such that

$$
\phi\left(b_{0}, \ldots, b_{n}\right)=0
$$

whenever $b_{i} \in V$ for each $i=0, \ldots, n$. It follows from the continuity of $p$ that there exists an open neighborhood $U$ of $x$ in $X$ with

$$
p(U) \subset V \text {. }
$$

Then we have 


$$
\left(p_{s}^{\sharp} \phi\right)\left(x_{0}, \ldots, x_{n}\right)=\phi\left(p x_{0}, \ldots, p x_{n}\right)=0
$$

whenever $x_{i} \in U$ for each $i=0, \ldots, n$. Hence $x$ is not in the support of $p_{s}^{\sharp} \phi$. Since $x$ is arbitrary, $p_{s}^{\#} \phi$ is of empty support. This and (4.3) prove that

$$
p_{s}^{\sharp}\left(A_{0}^{n}(B, G)\right) \subset A_{s}^{n}(X, G) \cap A_{0}^{n}(X, G) .
$$

Next, let $\psi \in A_{s}^{n}(X, G) \cap A_{0}^{n}(X, G)$ be arbitrarily given. By (4.3), there is an $n$-function $\phi \in A^{n}(B, G)$ such that $\phi=p_{s}^{*} \phi$. It remains to show that the support of $\phi$ is empty. Let $b \in B$ be any given point. Since $p$ maps $X$ onto $B$, there is a point $x \in X$ with $p x=b$. Since $\psi$ is of empty support, there is an open neighborhood $U$ of $x$ in $X$ such that

$$
\psi\left(x_{0}, \ldots, x_{n}\right)=0
$$

whenever $x_{i} \in U$ for each $i=0, \ldots, n$. Call

$$
V=p(U) \text {. }
$$

Since $p$ is an open map, $V$ is an open neighborhood of $b$ in $B$. Let $\left(b_{0}, \ldots, b_{n}\right)$ be any point in $B^{n+1}$ with $b_{i} \in V$ for each $i=0, \ldots, n$. Choose $n+1$ points $x_{0}, \ldots, x_{n}$ in $U$ such that $p x_{i}=b_{i}$ for each $i=0, \ldots, n$. Then we have

$$
\phi\left(b_{0}, \ldots, b_{n}\right)=\psi\left(x_{0}, \ldots, x_{n}\right)=0 .
$$

This proves that $b$ is not in the support of $\phi$. Since $b$ is arbitrary, the support of $\phi$ must be empty. This completes the proof of (4.5).

Since $p^{\#}$ maps $A_{0}^{n}(B, G)$ into $A_{0}^{n}(X, G)$ by (4.5), it induces a homomorphism

$$
p^{\#}: C^{n}(B, G) \rightarrow C^{n}(X, G) .
$$

By (4.3), $p^{\sharp}$ in (4.6) maps $C^{n}(B, G)$ into $C_{s}^{n}(X, G)$. Hence (4.6) may be written in the following more precise form

$$
p_{s}^{\sharp}: C^{n}(B, G) \rightarrow C_{s}^{n}(X, G) .
$$

(4.6) and (4.7) are connected by the following obvious relation

$$
\left\llcorner p_{i}^{\#}=p^{\#},\right.
$$

where $\iota: C_{j}^{\eta}(X, \mathrm{G}) \rightarrow C^{n}(X, G)$ denotes the inclusion homomorphism.

(4.9) Lemma. $p_{s}^{*}$ maps $C^{n}(B, G)$ isomorphically onto $C_{s}^{n}(X, G)$.

Proof. To prove that $p_{s}^{\sharp}$ maps $C^{n}(B, G)$ isomorphically into $C_{s}^{n}(X, G)$, let $c \in C^{n}(B, G)$ be any $n$-cochain of $B$ such that $p_{s}^{*} c=0$. Choose an $n$-function $\phi: B^{n+1} \rightarrow G$ which represents $c . \quad p_{s}^{*} c=0$ implies that $p_{s}^{*} \phi$ is of empiy support. By (4.3) and (4.5), this implies that the support of $\phi$ is empty. Hence $c=0$ and $p_{s}^{*}$ is an isomorphism.

To prove that $p_{s}^{*}$ maps $C^{n}(B, G)$ onto $C_{s}^{n}(X, G)$, let $d$ be any strongly invariant $n$-cochain of $X$ over $G$. Choose a $\psi \in A_{s}^{n}(X, G)$ which represents $d$. 
By (4.3), there is a $\phi \in A^{n}(B, G)$ such that $p_{s}^{*} i=\psi$. $\phi$ represents an $n$-cochain $c \in C^{n}(B . G)$ and obviously $p_{s}^{*} c=d$. This completes the proof of (4.9).

Since both $p^{\#}$ and $p_{s}^{*}$ commute with the coboundary operator $\delta$. they induce homomorphisms

$$
\begin{aligned}
& p^{*}: H^{\prime \prime}(B . G) \rightarrow H^{n}(X . G) \\
& p_{s}^{*}: H^{n}(B . G) \rightarrow H_{s}^{n}(X . G)
\end{aligned}
$$

for each integer $n \geq 0$. The relation (4.8) gives

$$
p_{s}^{*}=p^{*}
$$

The following theorem is an immediate consequence of (4.9).

THEOREM II. $p_{s}^{*}$ maps $H^{n}(B, G)$ isomorphically onto $H_{s}^{n}(X . G)$.

\section{The exact sequence}

Let us call

$$
d^{*}: H_{w}^{n}(X . G) \rightarrow H^{n+1}(B . G)
$$

the homomorphism defined by

$$
d^{*}=\left(p_{s}^{*}\right)^{-1} \partial^{*}
$$

'Then the following theorem is a consequence of the theorems I and $\mathrm{H}$ together with the relations $(4.12)$ and (5.1).

THEOREM III. The sequence of groups and homomorphisms

$$
H^{0}(B, G) \stackrel{p^{*}}{\rightarrow} \ldots \stackrel{d *}{\rightarrow} H^{n}(B . G) \stackrel{p^{*}}{\rightarrow} H^{n}(X . G)^{\pi^{*}} \rightarrow H_{w}^{n}(X, G) \stackrel{d *}{\rightarrow} H^{n+1}(B . G)^{p^{*}} \stackrel{\cdots}{\rightarrow}
$$

is exact.

\section{The canonical homomorphism $k_{x}^{*}$}

Let $x \in X$ be a given point. We are going to construct a canonical homomorphism

$$
k_{x}^{*}: H_{w}^{n}(X, G) \rightarrow H^{n}(Q, G)
$$

for each integer $n \geq 0$.

Let $\alpha \in H_{w}^{n}(X, G)$ be arbitrarily given. $\alpha$ is represented by a weakly residual $n$-cocycle $c_{w} \in Z_{w}^{n}(X, G)$ and $c_{w}$ itself is represented by an $n$-function $\phi \in A^{n}(X$. $G)$ such that

$$
\delta \phi=\xi+\eta, \quad \lessgtr \in A_{s}^{n+1}(X, G) . \quad \eta \in A_{0}^{n+1}(X, G) .
$$

We may assume that

$$
\phi(x \ldots \ldots x)=0
$$


In fact. if $\phi(x . \ldots x)=a \neq 0$, we define a strongly invariant $n$-function $\psi_{a} \in A_{s}^{n}(X$. $G)$ by taking

$$
\psi_{a}\left(x_{1} \ldots \ldots x_{n}\right)=a
$$

for each point $\left(x_{1} \ldots \ldots x_{n}\right)$ of $X^{n+1}$. Then, we replacc $\phi$ by $\phi-\psi_{a}$ which represerts the same weakly residual $n$-cocycle $c_{w}$ that $\phi$ does.

Now let us define an $n$-function $k^{*} \phi \in A^{n}(Q, G)$ of $Q$ over $G$ by taking

$$
\left(k^{\#} \phi\right)\left(q_{0}, \ldots q_{n}\right)=\phi\left(q_{0} x, \ldots, q_{n} x\right)
$$

for each point $\left(q_{0} \ldots, q_{n}\right)$ of $Q^{n+1}$.

(6.4) LemMA. The coboundary $\delta k^{*} \phi$ of $k^{*} \phi$ is of empty support.

Proof. Let $q$ be an arbitrary point in $Q$. It suffices to show that $q$ is not in the support of $j k^{*} \phi$. By 16.2 ). we have

$$
\grave{\phi} \phi=\xi+\gamma \text {. }
$$

where $\vdots \in A_{s}^{n+1}(X . G)$ and $\eta \in A_{0}^{n+1}(X, G)$. Since $\eta$ is of empty support there is an open neighborhood $U$ of the point $q x$ ih $X$ such that

$$
\eta\left(x_{0} \ldots \ldots x_{n}\right)=0
$$

whenever $x_{i} \in U$ for all $i=0 \ldots \ldots n$. Then there exists an open neighborhood $V$ of $q$ in $Q$ such that

$$
V x \subset U
$$

On the other hand. we have $\eta(x, \ldots, x)=0$. It follows that, for any point $\left(q_{1} \ldots \ldots q_{n+1}\right)$ of $Q^{n-2}$ such that $q_{i} \in V$ for all $i=0, \ldots, n+1$, we have

$$
\begin{aligned}
& \delta k^{*} \phi\left(q_{n} \ldots, q_{n+1}\right)=\sum_{i=0}^{n+1}(-1)^{i} k^{*} \phi\left(q_{0} \ldots . \hat{q}_{i}, \ldots, q_{n+1}\right) \\
& =\sum_{i=0}^{n-1}(-1)^{i} \phi\left(q_{0} x, \ldots \widehat{q_{i} x} \ldots . q_{n+1} x\right)=\delta \phi\left(q_{0} x, \ldots, q_{n+1} x\right) \\
& =\xi\left(q_{0} x, \ldots . q_{n+1} x\right)+\eta\left(q_{0} x, \ldots, q_{n+1} x\right)=\xi(x, \ldots, x) \\
& =\grave{\phi} \phi(x \ldots x)-\eta(x, \ldots x)=0 \text {. }
\end{aligned}
$$

This proves that $q$ is not in the support of $o k^{\sharp} \phi$ and hence completes the proof of (6.1).

By (6.4). the $n$-cochain $\left[k^{*} \phi\right] \in C^{\prime \prime}(Q, G)$ which contains the $n$-function $k^{*}$ : defined above is an $n$-cocycie of $Q$ over $G$ and hence it represents an elemen: $k_{:}^{*}(\alpha)$ of $H^{\prime \prime}(Q, G)$.

(6.5) Lemma. The element $k_{x}^{*}(\alpha)$ does not dopend on the choice of the in function $\phi \in A^{n}(X . G)$ which represents the given element $\alpha \in H_{w}^{n}\left(X . G^{\prime}\right.$.

Proof. First assume $n>0$. Let $\phi^{\prime}$ be any $n$-function which represents a ard such that $\phi^{\prime}(x \ldots \ldots x)=0$. Then 


$$
\phi^{\prime}-\phi=\delta \phi+\theta+\tau
$$

where $\psi \in A^{n ! 1}(X, G), \theta \in A_{s}^{n}(X, G)$ and $\tau \in A_{0}^{n}(X, G)$. Define an $(n-1)$-function $\zeta \in A^{n+1}(Q, G)$ of $Q$ over $G$ by taking

$$
\zeta\left(q_{1} \ldots, \ldots, q_{n-1}\right)=\psi\left(q_{0} x, \ldots, q_{n-1} x\right)-\psi(x, \ldots, x)
$$

for each point $\left(q_{n)} \ldots, q_{n-1}\right)$ of $Q^{n}$. In order to prove (6.5) for $n>0$, it suffices to show that

$$
k^{*} \psi^{\prime}-k^{*} \phi-\delta 5
$$

has empty support. Let $q$ be an arbitrary point in $Q$. Since the support of $\tau$ is empty, there is an open neighborhood $U$ of the point $q x$ in $X$ such that

$$
\tau\left(x_{0}, \ldots x_{n}\right)=0
$$

whenever $x_{i} \in U$ for all $i=0 \ldots \ldots n$. Let $V$ be an open neighborhood of $q$ in $Q$ such that

\section{$V x \subset U$}

Then, for each point $\left(q_{0}, \ldots q_{n}\right)$ of $Q^{n+1}$ with $q_{i} \in V$ for all $i=0, \ldots, n$, we have

$$
\begin{aligned}
\left(k^{\#} \phi^{\prime}-k^{*} \phi-\delta \zeta\right)\left(q_{0}, \ldots, q_{n}\right) \\
\quad=\left(\phi^{\prime}-\phi\right)\left(q_{0} x, \ldots, q_{n} x\right)-\delta \phi\left(q_{0} x, \ldots, q_{n} x\right)+\delta \phi(x, \ldots, x) \\
\quad=\theta\left(q_{1} x, \ldots, q_{n} x\right)+\tau\left(q_{0} x, \ldots, q_{n} x\right)+\delta \psi(x, \ldots, x) \\
\quad=\theta(x, \ldots, x)+\delta \psi(x, \ldots, x) \\
\quad=\phi^{\prime}(x, \ldots, x)-\phi(x, \ldots, x)=0 .
\end{aligned}
$$

Hence $q$ is not in the support of $k^{\sharp} \phi^{\prime}-k^{\sharp} \phi-\delta \zeta$. Since $q$ is arbitrary, this proves that the support of $k^{\sharp} \phi^{\prime}-k^{\#} \phi-\delta \zeta$ is empty.

It remains to dispose of the trivial case $n=0$. Let $\phi$ and $\phi^{\prime}$ be any two ()-functins which represent the same element $\alpha \in H_{w}^{0}(X, G)$ and such that $\left.\phi^{\prime} x\right)$ $=0=\phi^{\prime}(x)$. Since $A_{0}^{0}(X, G)=0$, we have $\phi^{\prime}-\phi \in A_{s}^{0}(X, G)$. In order to prove (6.5) for $n=0$, it suffices to show that $k^{\#} \phi^{\prime}-k^{\#} \phi=0$. Let $q$ be an arbitrary point in $Q$. Then we have

$$
\left(k^{*} \phi^{\prime}-k^{*} \phi\right)(q)=\left(\phi^{\prime}-\phi\right)(q x)=\left(\phi^{\prime}-\phi\right)(x)=0 .
$$

Since $q$ is arbitrary, we have $k^{*} \phi^{\prime}-k^{\#} \phi=0$. This completes the proof of $(5.5)$.

The correspondence $\alpha \rightarrow k_{x}^{*}(\alpha)$ obviously defines a homomorphism of $H_{w}^{n}(X$, $G)$ into $H^{n}(Q, G)$. This completes the construction of the canonical homomorpism (6.1).

\section{Relations between the canonical homomorphisms}

'THEOREM IV. If $Q$ is compact and if $x$ and $y$ are two points contained in a compact connected subset $K$ of $X$, then $k_{x}^{*}=k_{y}^{*}$. 
Proof. Let $n \geq 0$ be an arbitrary integer and $\alpha \in h_{w}^{\prime \prime}(X, G)$ be an arbitrary element. It is required to prove that

$$
k_{x}^{*}(\alpha)=k_{y}^{*}(\alpha) \text {. }
$$

The element $\alpha$ is represented by an $n$-function $\phi \in A^{n}(X, G)$ such that

$$
i \phi=\dot{\xi}+\eta, \quad \xi \in A_{s}^{n+1}(X, G), \quad \eta \in A_{0}^{n+1}(X, G) \text {. }
$$

According to the construction of the canonical homomorphism $k_{z}^{*}$ for an arbitrary point $z \in X$. the element $k_{z}^{*}(\alpha)$ of $H^{n}(Q, G)$ is represented by the $n$-function

$$
k_{z}^{*} p: Q^{n+1} \rightarrow G
$$

defined by

$$
\left(k_{z}^{*} \phi\right)\left(q_{1}, \ldots, q_{n}\right)=\phi\left(q_{0} z, \ldots, q_{n} z\right)-\phi(z, \ldots, z)
$$

for each point $\left(q_{0}, \ldots, q_{i}\right)$ of $Q^{n+1}$.

Now, for any two points a and $b$ of $X$ and any $(n+1)$-function $\psi \in A^{n+1}(X$, $G)$, let us define an $n$-function

$$
D_{u, h} \psi: Q^{n+1} \rightarrow G
$$

of $Q$ by taking

$$
\left(D_{a, b} \psi\right)\left(q_{0}, \ldots q_{n}\right)=\sum_{i=0}^{n}(-1)^{i} \psi\left(q_{0} a, \ldots q_{i} a, q_{i} b, \ldots q_{n} b\right)
$$

for each point $\left(q_{0}, \ldots q_{n}\right)$ of $Q^{n-1}$. Let $E_{\alpha, b} \psi$ denote the constant $n$-function of $Q$ defined by

$$
\left(E_{a, b} \psi\right)\left(q_{0}, \ldots q_{n}\right)=\left(D_{a, b} \psi\right)(e, \ldots e)
$$

for each point $\left(q_{11} \ldots \ldots q_{n}\right)$ of $Q^{n+1}$, where $e$ denotes the neutral element of $Q$. Since $\xi \in A_{\varsigma}^{n \cdot 1}(X, G)$. clearly we have

$$
D_{a, b} \stackrel{\xi}{\xi}=E_{a, b} \doteq .
$$

If $n>0$. direct calculation shows that

$$
\begin{aligned}
k_{b}^{\sharp} \phi-k_{a}^{\sharp} \phi & =\left(\delta D_{a, b} \phi+D_{a, b} \delta \phi\right)-\left(\delta E_{a, b} \phi+E_{a, b} \delta \phi\right) \\
& =\delta\left(D_{a, b} \phi-E_{a, b} \phi\right)+D_{a, b} \eta-E_{a, b} \eta
\end{aligned}
$$

since $\partial \phi=\hat{s}+\gamma_{j}$ and $D_{a, b} \hat{\xi}=E_{a, b} \hat{\xi}$. If $n=0$, then we have

$$
k_{b}^{\sharp} \phi-k_{a}^{\sharp} \phi=D_{a, b} \grave{\delta} \phi-E_{a, b} \delta \phi=D_{a, b} \eta-E_{a, b} \eta \text {. }
$$

Since $\eta_{i}$ is of empty support, there exists for each point $z$ in $X$, an open neighborhood $U_{z}$ of $z$ in $X$ such that

$$
\eta\left(x_{0}, \ldots x_{n+1}\right)=0
$$

whenever $x_{i} \in U_{z}$ for each $i=0 \ldots \ldots n+1$. It follows from the simultaneous 
continuity of the operations of $Q$ on $X$ that, for each $z \in X$ and $w \in Q$ there exist an open neighborhood $V_{w}$ of $z$ in $X$ and an open neighborhood $W_{w}$ of $w$ in $Q$ such that

$$
W_{w} V_{w} \subset U_{w z} .
$$

Since $Q$ is compact. there are a finite number of points $w_{1}, \ldots, w_{m}$ such that the open sets

$$
\mathfrak{W}_{z}=\left\{W_{w_{1}}, \ldots, W_{w_{m}}\right\}
$$

form an open covering of $Q$. Call

$$
V_{z}=V_{w_{1}} \cap \ldots \cap V_{w_{m}}
$$

Then $V_{z}$ is an open neighborhood of $z$ in $X$.

Now let $a$ and $b$ be any two points in $V_{z}$. We are going to show that both $D_{a, a} \eta$ and $E_{a, b} \eta$ are of empty supports. Let $q$ be an arbitrary point in $Q$. Choose an open set $W_{w_{j}}$ from the covering $\mathfrak{W}_{z}$ which contains $q$. Then we have

$$
W_{w_{j}} V_{z} \subset U_{w_{j}} z \text {. }
$$

Let $\left(q_{0}, \ldots, q_{n}\right]$ be any point of $Q^{n+1}$ such that $q_{i} \in W_{w_{j}}$ for each $i=0, \ldots$, n. Then the points

$$
q_{0} a, \ldots, q_{n} a, q_{0} b, \ldots, q_{n} b
$$

are all contained in $U_{w_{j}} z$. Hence we have

$$
\left(D_{a, b} \eta\right)\left(q_{0}, \ldots, q_{n}\right)=\sum_{i=0}^{n}(-1)^{i} \eta\left(q_{0} a, \ldots, q_{i} a, q_{i} b, \ldots, q_{n} b\right)=0 .
$$

This proves that $q$ is not in the support of $D_{a, b} \eta$. Since $q$ is arbitrary, the support of $D_{a, b} \eta$ must be empiy. This implies that

$$
\left(E_{a, b} \eta\right)\left(q_{0}, \ldots, q_{n}\right)=\left(D_{a, b \eta}\right)(e, \ldots, e)=0
$$

for every point $\left(q_{0}, \ldots, q_{n}\right)$ of $Q^{\eta+1}$. That is to say, $E_{a, b} \eta=0$ and hence $E_{a, b} \eta$ is of empty support. Then it follows from (7.1) and (7.2) that

$$
k_{a}^{*}(\alpha)=k_{b}^{*}(\alpha) \text {. }
$$

Since $x$ and $y$ are contained in a compact connected subset $K$ of $X$, there exist a finite number of points $z_{1}, \ldots, z_{r}$ of $X$ such that $x \in V_{z_{1}}, y \in V_{z_{r}}$, and the intersection $V_{z_{i}} \cap V_{z_{i+1}}$ is nonvoid for every $i=1, \ldots, r-1$. Choose a point $t_{i}$ from $V_{z_{i}} \cap V_{z_{i+1}}$ for each $i=1, \ldots, r-1$ and call $t_{0}=x t_{r}=y$. Thus we obtain a finite sequence of points

$$
x=t_{0}, t_{1}, \ldots, t_{r-1}, t_{r}=y
$$

such that $V_{z_{1}}$ contains $t_{i-1}$ and $t_{i}$ for each $i=1, \ldots, r$. By (7.3), this implies that

$$
k_{t_{i-1}}^{*}(\alpha)=k_{t_{i}}^{*}(\alpha)
$$


for each $i=1, \ldots, r$. Hence we obtain $k_{x}^{*}(\alpha)=k_{y}^{*}(\alpha)$. This completes the proof of Theorem IV.

A topological space $X$ is said to be compactly connected if every pair of points $x$ and $y$ of $X$ are contained in some compact connected subset of $X$. Compact connected spaces and arcwise connected spaces are examples of compactly connected spaces.

The following theorem is an immediate consequence of Theorem IV.

THEOREM V. If a compact transformation group $Q$ operates on a compacti.y connected topolcgical space $X$, then the canonical homomorphism $k_{x}^{*}$ does not depend on the choice of the basic point $x \in X$ and hence it may be denoted by

$$
k^{*}: H_{w}^{n}(X, G) \rightarrow H^{n}(\mathrm{Q}, G) .
$$

\section{BIBLIOGRAPHY}

[1] E. H. Spanier, Cohomology theory for general spaces, Ann. of Math. (2), Vol. 49 (1948), pp. 407-427.

2] J. L. Kelly and E. Pitcher, Exact homomorphism sequences in homology theory, Ann. of Math. (2), Vol. 48 (1947), pp. 682-709.

Tulane University and

The Institute for Advanced Study 